\title{
Influence of the shroud leading edge shape on the axial-fan noise
}

\author{
David Kohout ${ }^{1}$, Tomáš Hyhlík ${ }^{2}$ \\ Czech Technical University at Prague - Faculty of the Mechanical Engineering \\ Department of Fluid Dynamics and Thermodynamics \\ Technická 4, Prague, Czech Republic \\ david.kohout@fs.cvut.cz
}

\begin{abstract}
The paper is focused on the noise reduction of the low-pressure axial fan by the modification of the shroud leading edge shape. The CFD and CAA simulations of the fan are performed on commercial software PowerFLOW based on the Lattice Boltzmann method. The propagation of the acoustics waves to the far-field was carried out by the Ffowcs-Williams \& Hawkings acoustics analogy. The far-field acoustic data from the simulation matches with the results from the ISO measurement in the semi-anechoic chamber. Direct acoustics results of the near-field capture dominant noise sources of the cooling axial fan in blade tip area. Vortexes produced by the flow separation from the shroud inlet edge are carried by the rotating fan blade. The interaction of these flow structures with the blade tip surface produces strong subharmonic component in the acoustic spectra with lower frequency compared to blade passing frequency. Four different shrouds geometry modifications are analyzed. The modification of the shape eliminates subharmonic component of the fan sound spectra and reduces overall fan noise.
\end{abstract}

Keywords: Computational Aeroacoustics, Axial-fan, Lattice Boltzmann method, Subharmonic-hump

\section{Introduction}

Low pressure axial fans are commonly used in the large number of engineering applications related to the cooling system. The increase of the machine power caused higher requirements for the cooling package in automotive, railway, aircraft industry and construction machinery. The introduction of the noise restriction by the European union leads to effort for better understanding of the noise generating mechanism and to find the optimal condition for the cooling system. The major part of well-designed cooling system is the axial fan with optimal operating conditions such as high flow rate and low noise emission. Therefore, many studies focused on the description of noise generating mechanism of axial fan were presented.

The components of acoustic spectra were separated to broadband and discrete tonal noise. The random pressure fluctuation on blade surface caused by the turbulent boundary layer, ingestion turbulence, trailing edge and vortex shedding generate broadband noise [1,2]. The periodical blade forces induce the discreate noise. The characteristic tonal component of the low-pleasure axial fan is blade passing frequency related to the number of blades and rotation speed [3]. However, the dominant influence on the noise emission and efficiency of the axial fan has the tip gap region. Tip clearance vortexes produced by the pressure difference between the pressure and suction side are source of broadband noise. The following articles [4,5] focused on the tip leakage flow show "quasi-tonal structures" subharmonic hump in the noise spectra generated by coherent flow structures in tip gap region. This flow structures are carried by the fan blades and interact with them. Because of the lower rotation speed of these structures the generated noise has lower frequency than the blade passing frequency.

The presented study analyses the influence of the shroud leading shape on the axial fan noise and generated tonal components. The unsteady flow field around the axial fan is computed by the unconventional Lattice Boltzmann method. This data is used for better understanding of the flow topology in the tip gap region, which induces the subharmonic tonal noise component. The Ffowcs-Williams and Hawkings acoustic analogy is used for the propagation of the acoustics signal to far-field. The ISO measurement in the semi-anechoic chamber is performed to validate far-field acoustic data from the simulation.

\section{Experiment}

The acoustics measurements of the axial fan were performed in the semi-anechoic chamber. This chamber meets the criteria (ISO 3744, 3745) for determination of the sound power level of noise source from sound pressure level. The sound 
emission measurement was performed by six $1 / 2$ inch free field microphones type $4189-\mathrm{A}-021$ (Brüel \& Kjaer) placed on the hemispherical surface with 10 meters radius. The chamber's scheme is shown in Figure 1(a). Dewesoft SIRIUS module was used for data acquisition. The sampling frequency of the measured acoustics signal was $\mathrm{f}_{\mathrm{s}}=50 \mathrm{kHz}$. The background noise in the semi-anechoic chamber was $25 \mathrm{~dB}(\mathrm{~A})$.

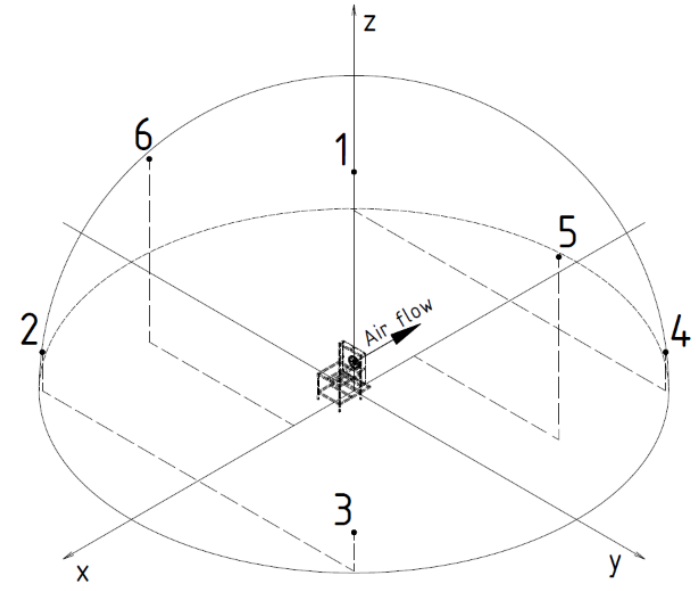

(a)

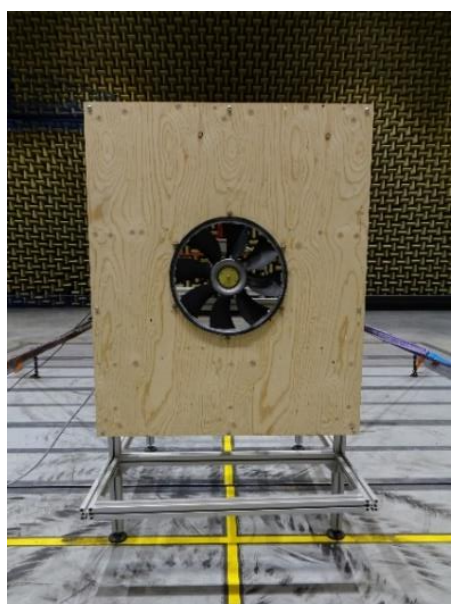

(b)

Figure 1: (a) - Scheme of the semi-anechoic chamber with microphones positions, (b) - Picture of the Fan test stand

The fan test stand was designed for the validation of the numerical aeroacoustics results by experiment. The axial fan was powered by electric motor, which was mounted on the aluminum construction. The suction side and exhaust side of the fan were separated by the rigid plywood desk. Main requirements were low influence of the construction and high rigidity of the construction, which is improved by fastening of the construction to the floor. The eight-blade axial fan was ducted in the shroud. The axial fan parameters are fan diameter $\mathrm{d}=390 \mathrm{~mm}$, hub diameter $\mathrm{d}_{\mathrm{h}}=134 \mathrm{~mm}$, blade skew $\alpha=$ $35^{\circ}$, tip clearance $\mathrm{s}=5 \mathrm{~mm}$. The suction side of the axial fan extends $3 / 4$ to full projected width into the shroud, which is showed in Figure 2. These parameters were set up close to parameters, which were used in cooling package of the small excavator except the smaller tip clearance. The fan speed for verification of the simulation was set up at $2455 \mathrm{rpm}$. The measurement was performed for four different shroud leading edge shape modifications:

- Shroud I - default shroud variant with leading edge thickness $5 \mathrm{~mm}$

- Shroud II - shroud with rounded leading edge

- Shroud III - shroud with sharped leading edge

- Shroud IV - shroud with inlet radius $40 \mathrm{~mm}$

The purpose of this modifications was to improve the inflow condition and reduce vortex structures in blade tip area related to the subharmonic hump. The designed shroud leading edge variants were based on the conclusions from the previous work [18]. The first two variants (shroud II and III) were based on the small geometric adjustments derived from the default variant. The last modification (shroud IV) sets the ideal inflow condition to compare with the previous variants. 

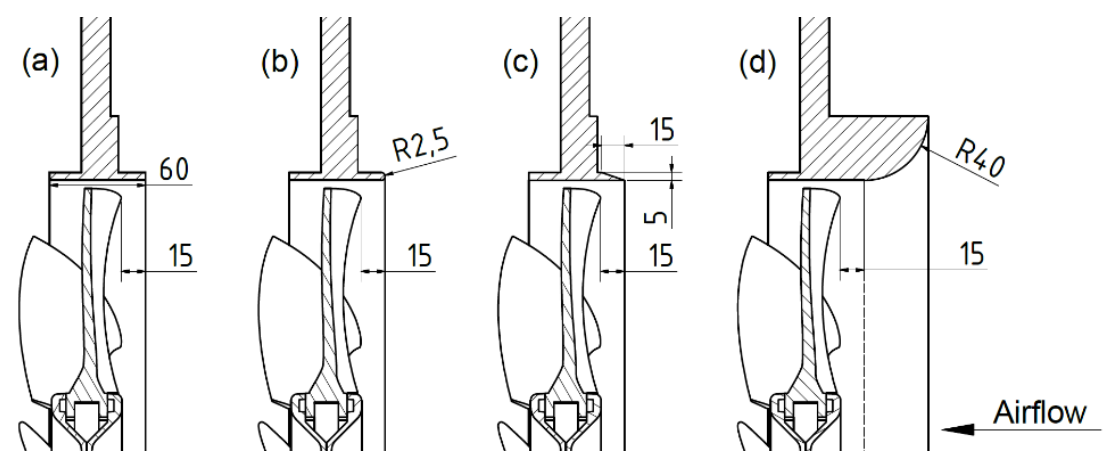

Figure 2: The Shroud shape variants: (a) shroud I (b) shroud II (c) shroud III (d) shroud IV

\section{Numerical simulation}

The hybrid CFD/FWH approach was used for the numerical computation of the acoustics field around the rotating axial fan. The CFD PowerFLOW solver is based on the unconventional Lattice Boltzmann Method (LBM). The LBM predicts the macroscopic behavior of the fluid dynamics based on the solution of mesoscopic kinetic equation. Unlike conventional CFD methods are LBM naturally transient, explicit and compressible.

\subsection{Lattice Boltzmann method}

The LBM describes the movement of the particles through the lattice grid by the distribution function $f_{i}$. PowerFLOW software uses the volumetric cubic lattice grid with the Variable Resolution strategy. The lattice element size increases with each VR twice. The distribution function expresses the number of the particles at time in defined volume with actual velocity. The movement of the particles is separated to the streaming and collision step. Streaming of the particles is fixed to the discrete i-velocity direction from the node of the lattice grid. The rate of the particles collisions is represented by collision operator, which is approximated by simplified models. PowerFLOW has incorporated the BGK model. This simplified collision model contains the relaxation time, which define time to reach equilibrium distribution function. The macroscopic quantities such as fluid density and fluid velocity are calculated from the summation of the distribution function. Detailed description of the LBM is in the following literature [6-9].

Turbulent flow is fully resolved in the fundamental form of the LBM. This approach is useful for detailed simulation of turbulent flows. However, direct simulation is restricted only for low Reynolds number due to the high computing demands. For this reason, are used the unresolved scales implemented by Large Eddy simulation (LES). The relaxation time was substituted by effective turbulent relaxation time that is derived from systematic renormalization group procedure. [10,11] The LBM method in combination with RNG k- $\varepsilon$ turbulent model is implemented in PowerFLOW software as Very Large Eddy simulation (VLES). The wall function is added to lower requirement for the mesh resolution near the wall. [12].

The rotation of the fan geometry was simulated by the Local reference frame (LRF) domain. The 3-D computational domain is divided into two parts. The coordinate system of the rotating geometry was "body-fixed". The outer part of the domain had the "ground-fixed" coordinate system. The interface between these regions connecting the separated mesh regions is called "sliding-mesh". The interface should not disturb the flow field around. The implementation of LRF in LBM is described in the following literature. [13]

\subsection{Acoustics approach}

The computational aeroacoustics gives two approaches to the computing of the acoustics field. The direct methods solve the unsteady flow and weak acoustics pressure fluctuation. The requirements are the high precession of the CFD method with grid and time refinement, because of the resolution of different scales of aerodynamic and acoustics values. Unlike the conventional solver based on Navier-Stokes equation the LBM has better assumptions for the acoustic 
simulation. The comparison of these methods for the standing planar wave shows lower dissipative behavior of the LBM. [14]

The hybrid approach is used in this paper for the acoustic far-field calculation. The PowerACOUSTICS software has implemented the Ffowcs-Williams and Hawkings (FW-H) analogy based on the Farrasat's 1A formulation of the FW-H equation $[15,16]$. This modification of $\mathrm{FW}-\mathrm{H}$ is commonly used for turbomachinery application. The acoustic waves are propagated from the permeable surface surrounding the rotating geometry region to microphones located in far-field region. The quadrupole noise sources are neglected. Acoustics waves reflection from the walls was considered. The implementation FWH to LBM (PowerFLOW) describe following literature [17]

\subsection{Simulation setup}

The computational domain with the fan test stand geometry showed in the Figure 3. corresponds with the presented experimental setup. Because of the high computational cost, three of four shroud variants were computed: shroud I, shroud III, shroud IV. The rotation of the axial fan is modelled by the LRF domain and the rotation speed is the same as in measurement $(2455 \mathrm{rpm})$. The characteristic axial-fan parameters are mentioned above. Pressure boundary conditions were set for the walls and ceiling of the computational domain. The floor was set as rigid perfectly reflective wall. The acoustic walls of the semi-anechoic chamber used to adjust free-field acoustic field were simulated by the sponge layers. Reference pressure in the domain was $101325 \mathrm{~Pa}$ and the reference temperature $\mathrm{T}=25^{\circ} \mathrm{C}$. The real Mach number for this simulation is equal $\mathrm{Ma}=0,142$.

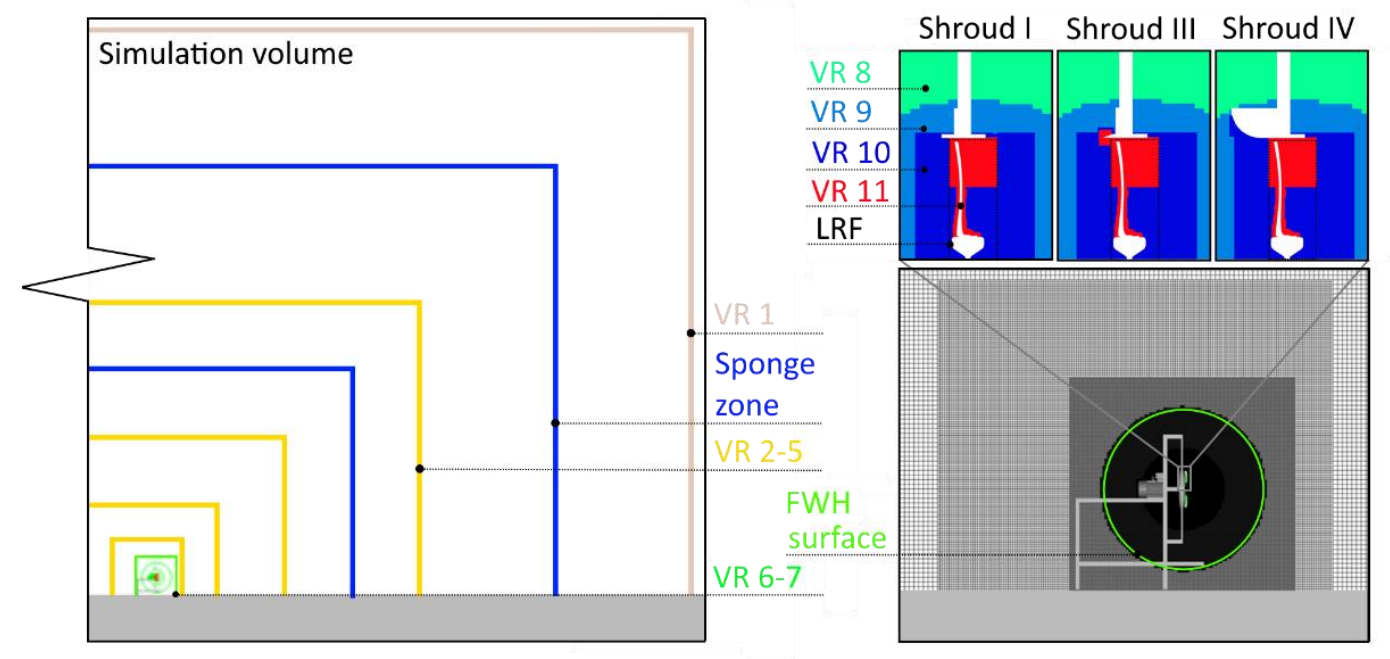

Figure 3: Scheme of the VR regions, Sponge zones, LRF zone, FWH surface in the simulation volume

The grid resolution in the computational domain defined by the VR is showed in the Figure 3 . The smallest element size around the fan blade and tip gap in VR11 was $\Delta \mathrm{x}=0,4 \mathrm{~mm}$. This resolution corresponds with 975 voxels per the fan diameter and 12 voxels across the tip clearance. The generated mesh has $159 \times 10^{6}$ voxels. The physical simulation time is equal to 20 fan revolutions. The flow and acoustics values are stored after 6 fan revolutions, when they are stabilized. The physical timestep is equal $\Delta \mathrm{t}=6,403 \times 10^{-7}$.

\section{Results and discussion}

The first part of the comparison among the all shroud leading edge shape modifications is based on the measured acoustic data from 6 microphones. The sound pressure level spectrum averaged among all microphones for each variant is compared in Figure 4. The acoustics spectrum is calculated for the 20 second length signal and the frequency resolution is $\Delta \mathrm{f}=2 \mathrm{~Hz}$. The Hanning window is applied with 50\% overlapping. The frequency range [70-2500] Hz contains the major noise sources of the axial fan. Blade passing frequency (BPF) of the measured axial fan is equal $327 \mathrm{~Hz}$. As can be seen in 
the Figure 4 (a) this tonal component is almost not visible for the first three variants (shroud I-III). This can be caused by the clean inflow conditions or this tonal component is covered by the broadband noise.

The Dominant peak is around $233 \mathrm{~Hz}$ and corresponding with the broadband humps. The subharmonic humps were presented in the following papers $[4,5,6]$. The frequency of this component is related to the blade passing frequency and it is approximately $\mathrm{f}_{\mathrm{SH}} \approx 0,7 \mathrm{f}_{\mathrm{BPF}} \approx 0.7 \times 327,3=229 \mathrm{~Hz}$. The noise generated mechanism is associated with the coherent flow structures in the tip gap region. Detailed description of the noise generating mechanism is shown by the acoustics pressure. The acoustics pressure is obtained from the timed derivation of the pressure field. The values of the acoustic pressure for the shroud I showed on the cut plane on the suction side of the fan and filtered for the frequency range around the subharmonic hump and BPF are shown in the Figure 4 (b). Unlike the BPF the subharmonic hump is generating by the strong pressure fluctuation in the tip gap. The BPF is representing by strong pressure fluctuation along the fan blade. The big difference in the acoustic spectrum can be seen in the last variant (shroud IV). The better inflow conditions decrease the broadband noise and remove the subharmonic component. The tonal component BPF is dominant and its harmonics are visible.

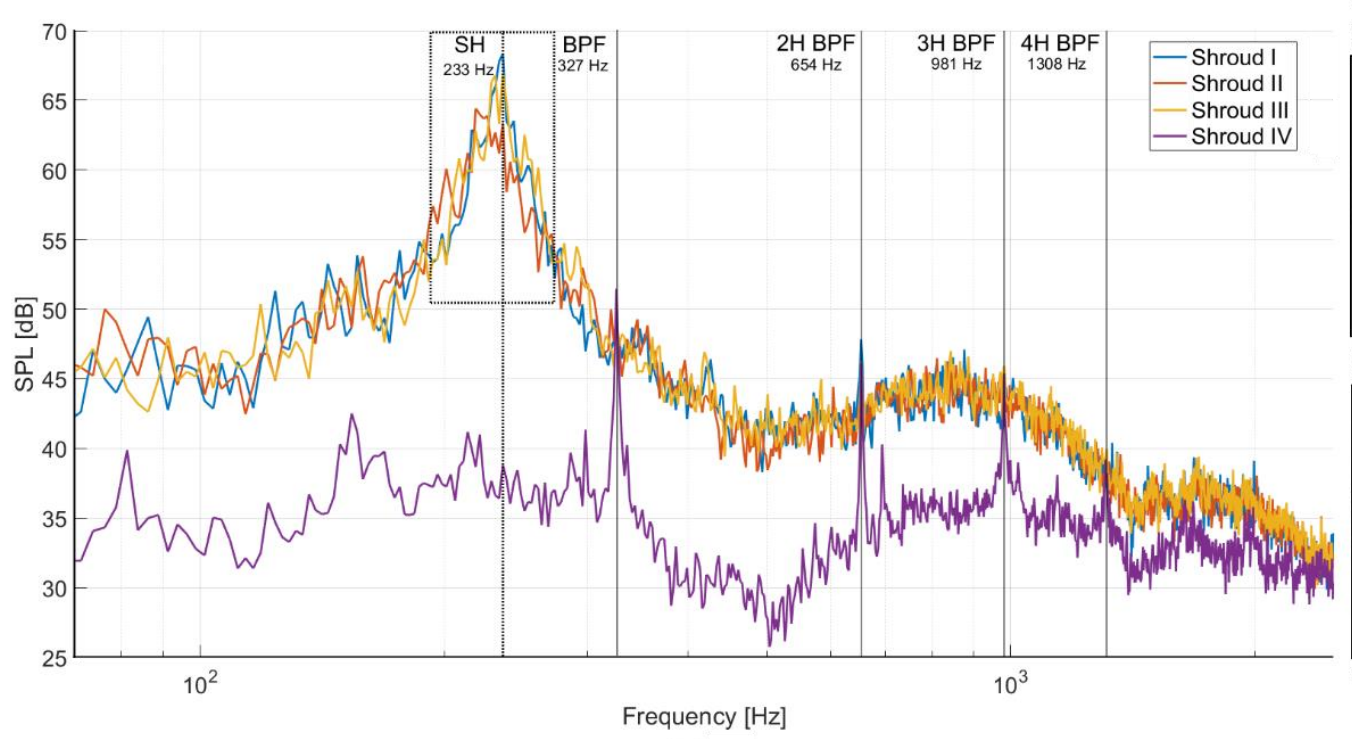

(a)
Shroud I

Band pass filter $(220-280 \mathrm{~Hz})$

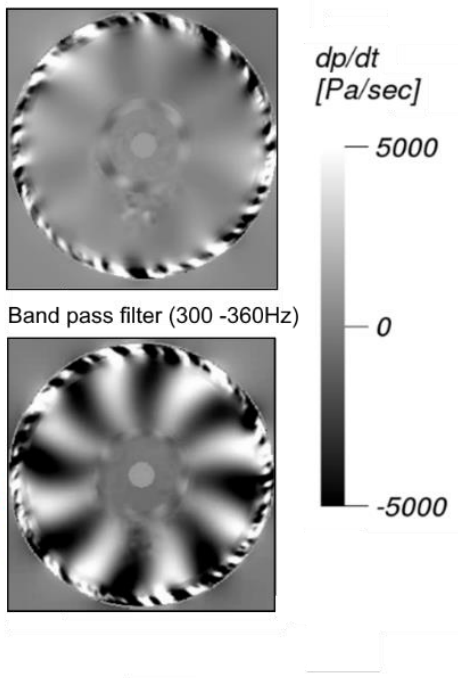

(b)

Figure 4: (a) Sound power spectrum from the measurement for each shroud leading edge shape variant (b) Band pass filters $(220-280 \mathrm{~Hz})$ and $(300-360 \mathrm{~Hz})$ of the acoustics pressure showed on the suction side of the axial fan for Shroud I

The noise reduction of each shroud leading edge shape modification is shown at the overall sound pressure level values $\left(\right.$ OASPL $_{\text {Shroud I }}=77,9 \mathrm{~dB}$; OASPL Shroud III $=76,9 \mathrm{~dB}$; OASPL Shroud IV $\left._{1}=64,7 \mathrm{~dB}\right)$. The least effect of the edge modification on the generated noise has the shroud II with the rounded edge. The sharp edge decreases the overall noise by $1 \mathrm{~dB}$. The maximal sound pressure level of subharmonic hump in default variant was decreased by the shroud II by $1,5 \mathrm{~dB}$ and shroud III by $4 \mathrm{~dB}$. Unlike these small design changes the last shroud with the inlet radius decreases the overall noise by $13,2 \mathrm{~dB}$. The noise reduction by this modification was predicted and is caused by the removing of the subharmonic humps. On the other hand, the blade sound pressure level on the BPF was increased by $4 \mathrm{~dB}$.

The acoustics results computed by the FWH acoustics analogy from the permeable surface pictured in the Figure 3 are validated by the results of the ISO measurement in the semi-anechoic chamber. The position of the microphone in the simulation is preserved the same as in the experiment. The spectral analysis parameters were unified to compare the results. The length of the signal from the simulation is 0.39 second and frequency resolution is $\Delta \mathrm{f}=4 \mathrm{~Hz}$. The sound presure level specrum for computed variants is shown in Figure 5. Time varying effect of the sound source for the measurement is shown in Figure 5 by the grey area. This interval represents the range of the acoustics values for diferent 
segment of the measurement. The segment length is same as for the simulatin 0.39 seconds. Sound pressure level spectrum was calculated for 200 different time segments.
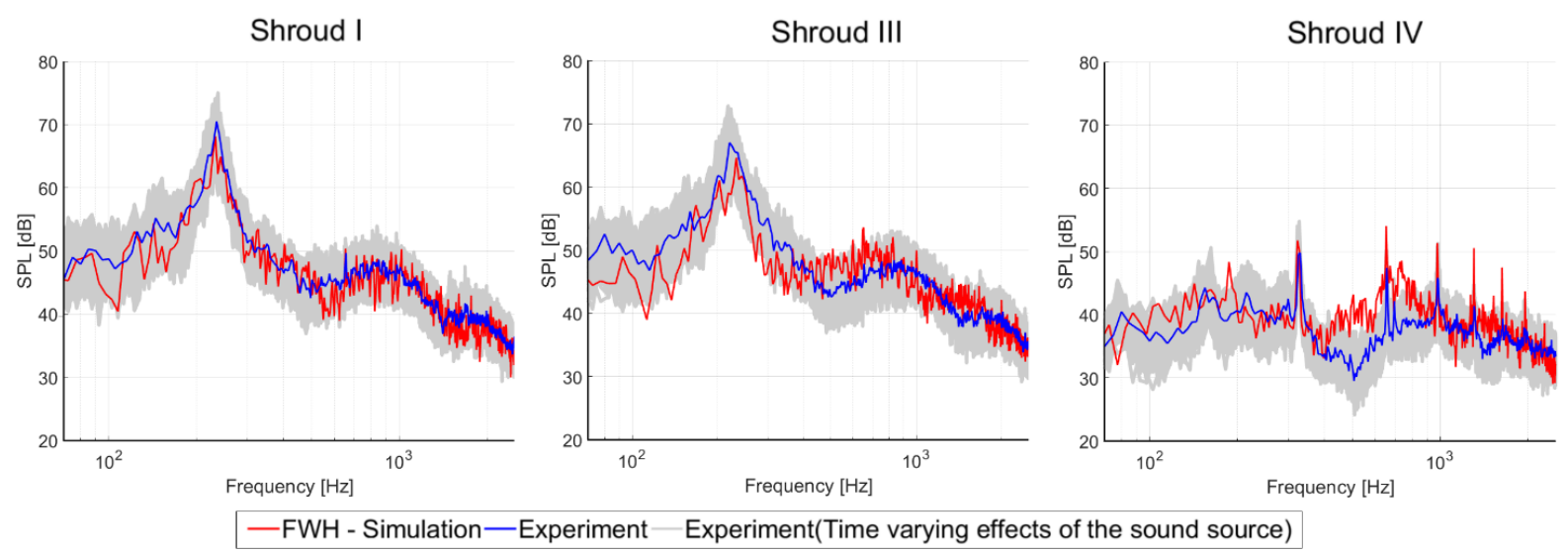

Figure 5: Validation of the sound pressure level spectrum calculated with the FWH acoustic analogy by the measured data for Shroud I, Shroud III, Shroud IV variant

The simulations for shroud I and III well predict the frequency range and amplitude of the subharmonic hump. The simulation results of the shroud III diverge more from the experiment than for shroud I. The last simulation of the shroud IV variant well predicted the BPF and its harmonics, but the simulation has higher amplitude of its harmonics than measurement. The values of the OASPL from the simulation are: OASPL Shroud I (Sim) $_{1}=76,1 \mathrm{~dB}$; OASPL Shroud III (Sim) $_{\text {On }}=74,8$ $\mathrm{dB}$; OASPL Shroud IV (Sim) $_{\text {S }}=67,5 \mathrm{~dB}$. The difference of the OASPL between the simulation and experiment is for Shroud I 1,8 $\mathrm{dB}$, Shroud III 2,1 dB and Shroud IV 2,8 dB.

The impact of the shroud leading edge shape modification on the aerodynamics performance of the axial fan is based on data from the simulation. The mass flow rate is measured on the surface placed on the suction side of the fan. The computed mass flow rate for the shroud I is $Q_{\text {Shroud I }}=2,45 \mathrm{~kg} / \mathrm{s}$, $Q_{\text {Shroud III }}=2,47 \mathrm{~kg} / \mathrm{s}$ and $Q_{\text {Shroud IV }}=2,73 \mathrm{~kg} / \mathrm{s}$. The mass flow rate for shroud I and III is the same and for the shroud IV increases by $11.6 \%$. To compare inflow condition and flow field around the axial fan the iso surface of $\lambda 2$ - criterion colored by vorticity magnitude is used. As can be seen in Figure 6 the shroud I and III is almost the same, more vortices separated from the shroud leading edge is cause by finer grid resolution around the edge.

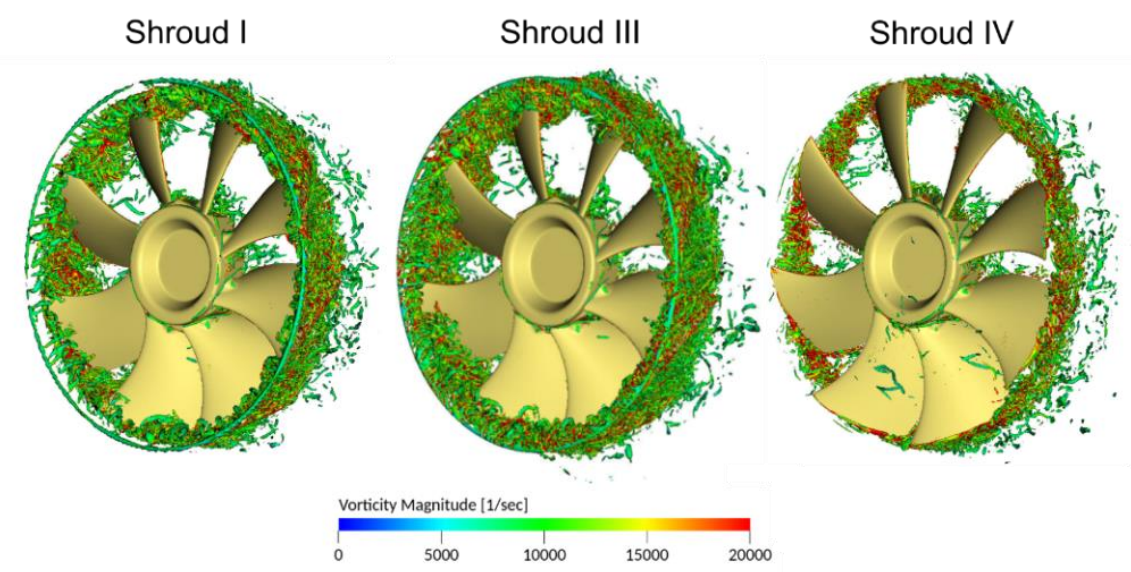

Figure 6: The turbulent flow structures showed by the $\lambda 2$ - criterion $\left(\lambda 2=-10^{7}\right)$ colored by the vorticity magnitude for Shroud I, Shroud III, Shroud IV variant 
It is obvious from this comparison that the generated subharmonic hump is caused by vortexes produced by the flow separation from the shroud leading edge. The shroud IV variant with the big inlet radius has no flow structures on the suction side only the tip leakage vortexes.

\section{Conclusion}

The influence of the shroud leading edge shape on the axial fan noise was analysed for four different geometry variants by the CFD/CAA simulation and the measurement in the semi-anechoic chamber. The results showed that the inflow condition can significantly increase the fan noise. The flow structures separated from the shroud leading edge generate the subharmonic hump $(233 \mathrm{~Hz})$, which was dominant in the spectra. Improvement of the inflow condition by the big inlet radius removed subharmonic hump and reduced the OASPL by $13,2 \mathrm{~dB}$. The mass flow rate increased by $11.6 \%$ against the default variant. The difference between the noise generating mechanism for the BPF and subharmonic hump was presented.

The acoustic spectrum computed from the FWH acoustics analogy well predicted the tonal components from the acoustic spectrum from the measurement. The OASPL difference between the experiment and the simulation was from the $1.8 \mathrm{~dB}$ up to $2.8 \mathrm{~dB}$.

\section{Acknowledgements}

This work was supported by the Grant Agency of the Czech Technical University in Prague, grant No. SGS20/112/OHK2/2T/12. I would also like to thank the Doosan Bobcat company especially Advance analysis department and Test department for providing the semi-anechoic chamber for the experiment and software from the Exa company for the CFD/CAA simulations.

\section{References}

[1] WRIGHT, S.E. The acoustic spectrum of axial flow machines. Journal of Sound and Vibration. 1976, 45(2), 165223. DOI: 10.1016/0022-460X(76)90596-4. ISSN 0022460X.

[2] SHARLAND, I.J. Sources of noise in axial flow fans. Journal of Sound and Vibration [online]. 1964, 1(3), $302-322$ [cit. 2020-04-23]. DOI: 10.1016/0022-460X(64)90068-9. ISSN 0022460X.

[3] L, Gutin. On the sound field of a rotating propeller: Über das Schallfeld einer rotierenden Luftschraube. 1. Washington, DC: National Advisory Committee for Aeronautics, 1948.

[4] MOREAU, Stéphane a Marlène SANJOSE. Sub-harmonic broadband humps and tip noise in low-speed ring fans. The Journal of the Acoustical Society of America [online]. 2016, 139(1), 118-127 [cit. 2020-04-23]. DOI: 10.1121/1.4939493. ISSN 0001-4966.

[5] MOGHADAM, S. M. Alavi, M. MEINKE a W. SCHRÖDER. Numerical Analysis of the Acoustic Field of a Ducted Axial Fan at Varying Tip Clearances. Acta Acustica united with Acustica. 2019, 105(1), 43-55. DOI: 10.3813/AAA.919286. ISSN 1610-1928.

[6] MOHAMAD, A. A. Lattice Boltzmann method: fundamentals and engineering applications with computer codes / A. A. Mohamad. New York: Springer, c2011. ISBN 978-0-85729-454-8.

[7] CHEN, H. Extended Boltzmann Kinetic Equation for Turbulent Flows. Science. 2003, 301(5633), 633-636. DOI: 10.1126/science.1085048. ISSN 0036-8075.

[8] AIDUN, Cyrus K. a Jonathan R. CLAUSEN. Lattice-Boltzmann Method for Complex Flows. Annual Review of Fluid Mechanics. 2010, 42(1), 439-472. DOI: 10.1146/annurev-fluid-121108-145519.ISSN 0066-4189.

[9] SUCCI, Sauro. The Lattice Boltzmann equation: for fluid dynamics and beyond. Oxford: Oxford University Press, 2013. ISBN 978-019-9679-249.

[10] TEIXEIRA, Christopher M. Incorporating Turbulence Models into the Lattice-Boltzmann Method. International Journal of Modern Physics C. 1998, 09(08), 1159-1175. DOI: 10.1142/S0129183198001060. ISSN 0129-1831.

[11] CHEN, HUDONG, STEVEN A. ORSZAG, ILYA STAROSELSKY a SAURO SUCCI. Expanded analogy between Boltzmann kinetic theory of fluids and turbulence. Journal of Fluid Mechanics. 1999, 519(1), 301-314. 
DOI:10.1017/S0022112004001211. ISSN 0022-1120.

[12] LAUNDER, B.E. a D.B. SPALDING. The numerical computation of turbulent flows. Computer Methods in Applied Mechanics and Engineering. 1974, 3(2), 269-289. DOI: 10.1016/0045-7825(74)90029-2.ISSN 00457825.

[13] ZHANG, Raoyang, Chenghai SUN, Yanbing LI, Rajani SATTI, Richard SHOCK, James HOCH a Hudong CHEN. Lattice Boltzmann Approach for Local Reference Frames. Communications in Computational Physics. 2011, 9(5), 1193-1205. DOI: 10.4208/cicp.021109.111110s. ISSN 18152406.

[14] MARIÉ, Simon, Denis RICOT a Pierre SAGAUT. Comparison between lattice Boltzmann method and NavierStokes high order schemes for computational aeroacoustics. Journal of Computational Physics. 2009, 228(4), 10561070. DOI: 10.1016/j.jcp.2008.10.021. ISSN 00219991.

[15] WILLIAMS, J. E. F. a D. L. HAWKINGS. Sound Generation by Turbulence and Surfaces in Arbitrary Motion. Philosophical Transactions of the Royal Society A: Mathematical, Physical and Engineering Sciences. 1969, 264(1151), 321-342. DOI: 10.1098/rsta.1969.0031. ISSN 1364-503X.

[16] Farassat, F. (2007) Derivation of Formulations 1 and 1A of Farassat. Tech. Rep. TM-2007-214853 NASA/TM2007-214853.

[17] BRÈS, Guillaume, Franck PÉROT a David FREED. A Ffowcs Williams - Hawkings Solver for Lattice-Boltzmann Based Computational Aeroacoustics. 16th AIAA/CEAS Aeroacoustics Conference. Reston, Virigina: American Institute of Aeronautics and Astronautics, 2010, 2010-06-07, -. DOI: 10.2514/6.2010-3711. ISBN 978-1-60086-9556.

[18] KOHOUT, David. Experimental and Numerical Analysis of Axial Fan Noise. Prague, Czech Republic, 2018. Master thesis. Czech Technical University at Prague - Faculty of the Mechanical Engineering. 